\title{
Short-term outcomes of down-referral in provision of paediatric antiretroviral therapy at Red Cross War Memorial Children's Hospital, Cape Town, South Africa: A retrospective cohort study
}

\author{
J Copelyn, ${ }^{1}$ MBBS, FC Paed (SA), MMed; P Apolles, ${ }^{2} \mathrm{RN}$; M-A Davies, ${ }^{3} \mathrm{MB}$ ChB, MMed, FCPHM, PhD; \\ B Eley, ${ }^{2} \mathrm{MB}$ ChB, FC Paed (SA), BSc Hons

\begin{abstract}
${ }^{1}$ Department of Paediatrics and Child Health, Faculty of Health Sciences, University of Cape Town, South Africa
${ }^{2}$ Paediatric Infectious Diseases Unit, Red Cross War Memorial Children's Hospital and Department of Paediatrics and Child Health, Faculty of Health Sciences, University of Cape Town, South Africa

${ }^{3}$ Centre for Infectious Disease Epidemiology and Research, School of Public Health and Family Medicine, Faculty of Health Sciences, University of Cape Town, South Africa
\end{abstract}

Corresponding author: J Copelyn (julie_copelyn@yahoo.com)

\begin{abstract}
Background. The large scale-up of paediatric HIV care necessitated down-referral of many children receiving antiretroviral therapy (ART) from Red Cross War Memorial Children's Hospital (RCWMCH), Cape Town, South Africa. Few published data exist on the outcomes of these children.

Objectives. To assess outcomes of children receiving ART in the first 12 months after down-referral to primary healthcare (PHC) clinics and identify determinants of successful down-referral.

Methods. A retrospective cohort study of children $<15$ years of age who initiated ART at RCWMCH and were subsequently down-referred to one of two PHC clinics between January 2006 and December 2012 was completed. Baseline characteristics of patients and caregivers as well as CD4+ counts, viral loads (VLs) and weights were collected 6 and 12 months after down-referral. Outcomes included retention in care and viral suppression.

Results. Of 116 children down-referred to the two study PHC clinics, $81.9 \%$ arrived at the designated PHC clinic and a further $8.6 \%$ continued care at other clinics, the remaining $9.5 \%$ being lost to follow-up. Of those successfully down-referred, $11.4 \%$ took $>8$ weeks to present, possibly experiencing treatment interruption. At 12 months after down-referral, only $81.0 \%$ remained in care. No factors were associated with retention in care in multivariable analysis. For children who remained in care at the designated PHC clinics, the clinical and immunological gains achieved prior to down-referral were sustained through 12 months of follow-up, and $54.7 \%$ of this cohort had documented viral suppression at 12 months. However, if only children with VL results are considered, 75.9\% (41/54) were virally suppressed 12 months after down-referral. Conclusions. Down-referral of children on ART is complex, with risk of loss to follow-up and treatment interruption.
\end{abstract}

S Afr Med J 2018;108(5):432-438. DOI:10.7196/SAMJ.2018.v108i5.12855

The HIV/AIDS epidemic has had a dramatic impact on the health of South Africa (SA)'s children, contributing substantial morbidity and mortality. Without antiretroviral therapy (ART) it is estimated that $>50 \%$ of perinatally infected children will die before their second birthday, ${ }^{[1]}$ primarily as a result of rapid disease progression, early severe HIV disease or life-threatening co-infections. ${ }^{[2]}$ The SA national ART programme was launched in April 2004 and is now the biggest programme in the world, with $>3$ million people receiving ART, including an estimated 160000 children. Since its inception, the national ART programme has grown rapidly and resulted in substantial positive change. Through improvements in the prevention of mother-to-child transmission (PMTCT) programme, vertical transmission was reduced from $15 \%$ in 2009 to $4 \%$ in 2014, averting an estimated 370000 new childhood HIV infections. ${ }^{[3]}$ The 2013 national guideline ${ }^{[4]}$ further expanded paediatric access to ART by broadening initiation criteria to include all HIV-infected children aged $<5$ years. By 2014, almost half of all HIV-infected children in the country were accessing ART. While still significantly below target, this was an increase from just $11 \%$ in $2009 .{ }^{[3]}$
In the push for rapid scale-up of ART provision and improvement in ART coverage, the SA government adopted a public health approach to HIV care. Central to this approach are the two closely linked concepts of task shifting and decentralisation. Task shifting entails 'lower' cadres of healthcare providers, and sometimes non-professionals, taking over responsibilities previously held by doctors or 'higher' cadres of healthcare workers. ${ }^{[5]}$ Decentralising care includes moving care from tertiary referral centres to healthcare centres or clinics, and even beyond clinics into communities, closer to patients' places of residence. ${ }^{[6]}$ For many patients this has required a process of downreferral to sites with less specialised healthcare facilities. Progress with paediatric down-referral has been slow, however, in part owing to healthcare worker perceptions regarding the complexity of managing HIV-infected children.

The paediatric ART programme in the Western Cape Province, SA, has made considerable efforts to decentralise care of HIV-infected children. Between March 2004 and December 2015, the number of clinics in the province treating children with ART increased from 11 to 180 , and the percentage of children receiving ART from the three Cape Town tertiary hospitals declined from $78.4 \%$ to $14.7 \%^{[7}$ 
(and ART Monthly Enrolment Summaries, 2015, HAST (HIV \& AIDS, STI and TB) Directorate, Department of Health Western Cape - unpublished data). By 2007/2008 the ART coverage rate in the province had improved to $>95 \%$, well exceeding the national average of $37 \%{ }^{[8]}$ This was a consequence of both significant gains in PMTCT of HIV and expansion of the paediatric ART programme into primary healthcare (PHC) settings. This decentralised model of care necessitated down-referral of large numbers of children from tertiary academic hospitals to community-based clinics. Data on both the success of the down-referral process and the clinical outcomes of these children after down-referral are severely limited. In a recent study of transfer in adolescents receiving ART in the Western Cape, $>80 \%$ transferred successfully from tertiary care facilities to PHC clinics. ${ }^{[9]}$ In a further study of younger children receiving ART at physician-run PHC clinics, almost all of 270 children who had been down-referred from tertiary care in the Western Cape either sustained or achieved virological suppression over a median of 28 months of follow-up. ${ }^{[10]}$

\section{Objective}

To assess the success of the down-referral process and the clinical and ART outcomes in a cohort of young children, and to explore determinants of successful down-referral.

\section{Methods \\ Setting}

The study was conducted at Red Cross War Memorial Children's Hospital (RCWMCH) and two PHC clinics in Cape Town.

RCWMCH is a tertiary facility that serves as a referral centre for the paediatric population of the Western Cape and surrounding provinces. HIV-infected children have been accessing ART through this service since 1998. Since 2006, in accordance with the provincial framework for managing HIV-infected children, many children have been actively down-referred, mainly to level 1 paediatric HIV clinics in the Cape metropolitan area. Down-referral criteria have not been standardised, and while clinicians in the outpatient HIV clinic (OHC) are encouraged to down-refer stable patients with clinical and laboratory evidence of compliance and immune recovery (such as no opportunistic infections, suppressed viral load (VL) and recovering CD4+ count), decisions are made on an individual patient basis. Furthermore, as the provincial ART programme matured, down-referral of children initiated on ART during hospital admission began to occur at discharge from inpatient care. Down-referral is facilitated by clinicians telephonically arranging the first PHC clinic appointment and providing caregivers with a written clinical summary for clinic staff.

The two PHC study clinics fall within the immediate drainage area of RCWMCH and are $<10 \mathrm{~km}$ apart. One is a large, well-established clinic that has been providing a paediatric HIV service since 2003. In comparison, the other is a much smaller clinic, which only began its paediatric ART outpatient service in 2010. Both paediatric HIV clinics are medical officer led.

\section{Study design}

The hospital ART database was used to identify all children who initiated ART at RCWMCH and were subsequently down-referred to either of the two PHC clinics between 1 January 2006 and 31 December 2012.

Baseline characteristics of patients and their caregivers were retrospectively extracted from case record forms held by the infectious diseases clinic. Hospital folders were further reviewed to extract information relating to hospital admission diagnoses, length of follow-up at RCWMH and clinical and virological parameters prior to the point of down-referral. CD4+ percentage (CD4\%) and VLs prior to down-referral were accepted within a window period of 6 months.

At the PHC clinics, data were again retrospectively collected from patient folders, including the time between down-referral (last appointment at RCWMCH) and presentation at the PHC and outcomes at 6 and 12 months after down-referral. Measures included the patient's weight and CD4\% and VL results. For 6- and 12-month CD4\% and VL results, the closest measures within a window period of 4 - 8 and 9 - 15 months, respectively, were used.

For patients whose outcomes could not be established at the two designated PHC clinics, the Western Cape Provincial Health Data Centre (PHDC) searched available provincial electronic databases for data on clinic visits, HIV-related laboratory tests and antiretroviral drugs dispensed for these children during the 12-month period after down-referral. The PHDC collates information on individual patients using a unique health identifier (folder number) across multiple patient information systems in the province.

Anthropometric measurements such as weight and height were converted to gender-specific weight-for-age $z$-scores (WAZ), heightfor-age $z$-scores (HAZ) and weight-for-height $z$-scores (WHZ) scores, according to the 2000 Centers for Disease Control and Prevention (CDC) growth charts. ${ }^{[1]} \mathrm{CDC}$ charts were used because they covered the full age range of patients in the study cohort. Moderate underweight for age, stunting and wasting were defined as WAZ, HAZ and WHZ between -2 and $-3 z$-scores, $<-3$ being defined as severe underweight, stunting and wasting. Specific outcomes measured at 6 and 12 months after down-referral included mean WAZ, WAZ category, median $\mathrm{CD} 4 \%$, the proportion of children with severe immunodeficiency as defined by the World Health Organization (WHO), ${ }^{[12]}$ the proportion who were virologically suppressed (defined as a $\mathrm{VL}<400$ copies $/ \mathrm{mL}$ ), and at 12 months the proportions who remained in care or had been lost to follow-up (LTFU).

\section{Data analysis}

Data were collected on a standardised data capture sheet and inputted into Excel version 14.7.1 (Microsoft, USA). Data were analysed using Stata software (Stata/IC 13.0 for Windows, StataCorp USA). Means and standard deviations (SDs) or medians and interquartile ranges (IQRs) were used to describe normally and non-normally distributed data, respectively. Student's $t$-test was used to compare normally distributed data and the Wilcoxon sum-rank test was used for skewed data. Categorical data were compared using the $\chi^{2}$ test or Fisher's exact test, as appropriate.

Univariate analyses using the unadjusted risk ratio (RR) and 95\% confidence intervals (CIs) as well as multivariable logistic regression modelling were performed to identify potential factors associated with retention in care at PHC clinics 12 months after down-referral.

\section{Ethics considerations}

The study was approved by the Human Research Ethics Committee, Faculty of Health Sciences, University of Cape Town (ref. no. 246/2013) and the Western Cape Department of Health. Patient consent was not obtained because the data were collected and collated retrospectively. The study was completed in accordance with the Declaration of Helsinki.

\section{Results}

The hospital ART database search identified 119 children meeting the study inclusion criteria. On further examination, 3 children were excluded from the analysis; 1 had initiated ART prior to 
presentation at RCWMCH, and 2 were not actively down-referred but rather 'selfdown-referred' without the knowledge of attending clinicians. Data for the remaining 116 children, referred to as the study cohort, were analysed (Fig. 1).

\section{Baseline characteristics}

The majority of the study cohort (61.2\%) was female. The median age at ART initiation was 11 months, and $52.6 \%$ were $<12$ months of age. Moderate or severe wasting and stunting were present in $29.2 \%$ and $39.3 \%$ of participants, respectively. There were no statistically significant differences in baseline sociodemographic or anthropometric features between participants who were and those who were not retained in care (RIC) at 12 months after down-referral (Table 1).
The mother was the primary caregiver for the majority of participants (87.1\%). However, very few mothers (14.4\%) were taking ART themselves and only $38.7 \%$ had received any PMTCT prophylaxis. Compared with participants who were RIC, a greater proportion of those not RIC after down-referral were receiving support grants at ART initiation ( $80.0 \%$ v. $50.0 \%$, respectively.)

\section{HIV diagnosis and hospitalisation}

The majority of the study cohort (79/116, $68.1 \%$ ) had undiagnosed HIV infection prior to referral to RCWMCH. Only $19.8 \%$ were diagnosed at a PHC clinic, the remainder having been diagnosed at other hospitals or by private doctors. At the time of ART initiation, $90.2 \%$ of the study cohort had advanced clinical disease (WHO

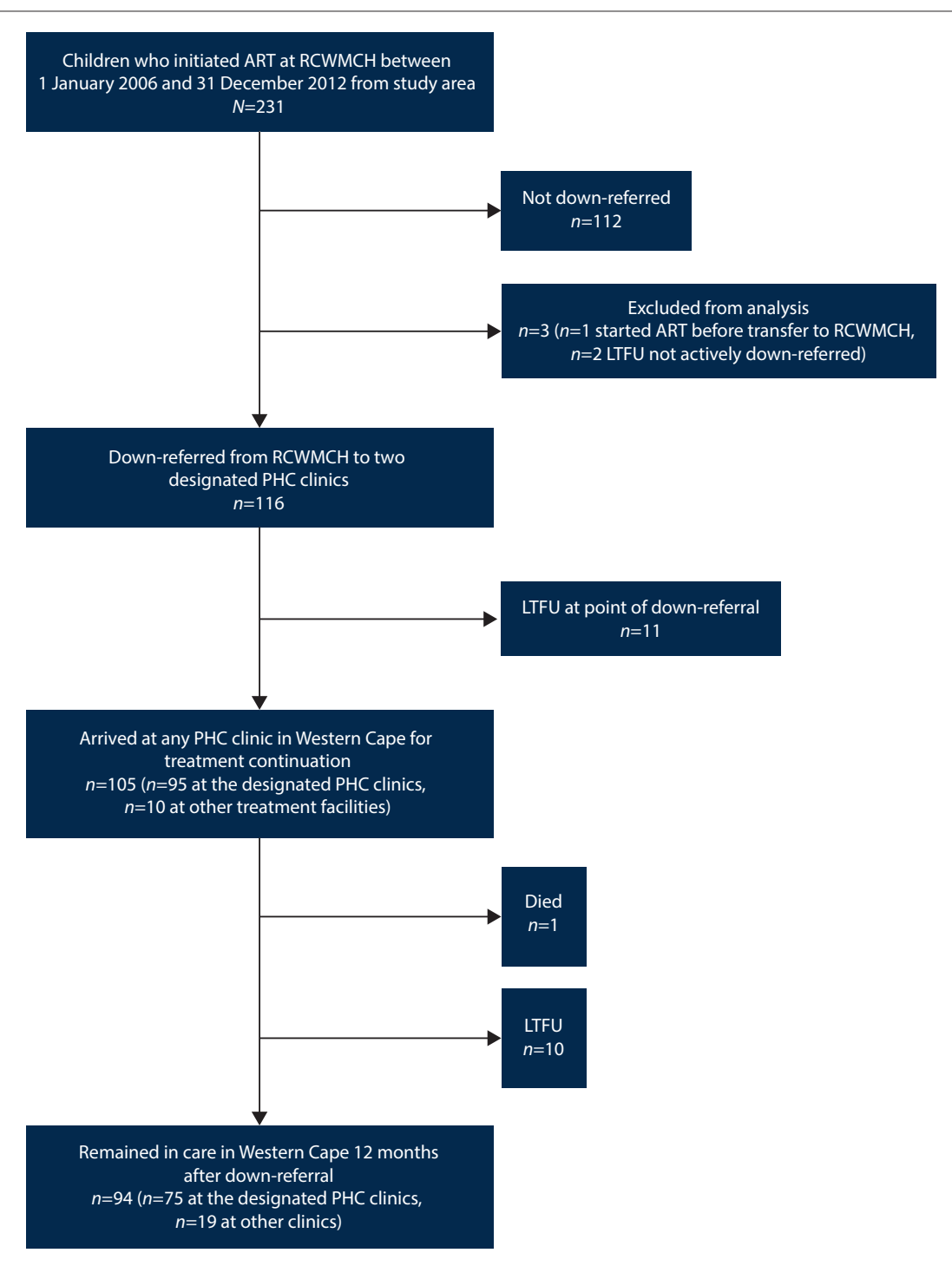

Fig. 1. Selection of study subjects and their progression during the study period. (ART = antiretroviral therapy; RCWMCH = Red Cross War Memorial Children's Hospital; LTFU = lost to follow-up; PHC = primary healthcare.) clinical stage 3 or 4 ), and the median (IQR) CD $4 \%$ was $18 \%(11-23)$. Treatment for coexistent tuberculosis (TB) was required by $18.1 \%$ of the study cohort, $23.8 \%(5 / 21)$ of whom were treated for extrapulmonary TB (Table 2).

Not all the children required hospitalisation; $13.9 \%$ were managed exclusively on an outpatient basis at the OHC. For the 99 children who were admitted to hospital, the mean (SD) length of stay was 19 (14) days and the most common admission diagnoses were acute lower respiratory tract infection (52.5\%) and diarrhoeal disease (36.4\%). Most of the children were managed in general medical or acute short-stay wards (70.2\%). However, $15.8 \%$ required admission to the intensive care unit. ART initiation occurred in an inpatient setting for $61.7 \%$ of the cohort, while the remaining $38.3 \%$ initiated ART in the OHC.

\section{ART management prior to down-referral}

All children in the study cohort were initiated on ART at RCWMCH. While the median number of days between HIV diagnosis and ART initiation was 42, this declined progressively from 414 days for those diagnosed before 2005 to 15 days for those diagnosed in 2011/2012, a decrease of $96 \%$.

Of the children who required hospitalisation, $21.2 \%$ (21/99) were initiated on ART and down-referred to PHC clinics to continue their treatment at the time of discharge. The remaining 95 children who were seen at the $\mathrm{OHC}$ at $\mathrm{RCWMCH}$ were followed up for a median of 320 days (IQR 189 - 685), with a mean (SD) number of 12 (8) clinic visits prior to down-referral, and $26.6 \%(25 / 94)$ of these children had at least one hospital admission during follow-up. The median age at down-referral was 26 months (IQR 12 - 52). At downreferral, $47.4 \%(55 / 116)$ of the cohort had a documented suppressed VL within the preceding 6 months. In a further $12.1 \%$ (14/116) no VL was done prior to downreferral, and in the remaining $40.6 \%$ (47/116) the last VL prior to down-referral was unsuppressed. Five children who had defaulted from ART while attending $\mathrm{RCWMCH}$ were down-referred to restart therapy at the referral clinic.

\section{Outcomes after down-referral}

Once down-referred, $90.6 \%(105 / 116)$ of children presented for treatment continuation, $81.9 \%(95 / 116)$ at the designated PHC clinic and a further $8.6 \%(10 / 116)$ at other 


\begin{tabular}{|c|c|c|c|c|}
\hline & Total $(N=116)^{*}$ & Not RIC $(n=22)^{*}$ & RIC $(n=94)$ & $p$-value ${ }^{\dagger}$ \\
\hline \multicolumn{5}{|l|}{ Sociodemographics } \\
\hline Male sex, $n(\%)$ & $45(38.8)$ & $8(36.4)$ & $37(39.4)$ & 0.795 \\
\hline Age at initiation, median (IQR) & $11(4-38)$ & $18(6-41)$ & $8(4-37)$ & $0.302^{\ddagger}$ \\
\hline Age group (years), $n$ (\%) & & & & 0.266 \\
\hline$<1$ & $61(52.6)$ & $9(40.9)$ & $52(55.3)$ & \\
\hline $1-5$ & $36(31.0)$ & $10(45.5)$ & $26(27.7)$ & \\
\hline$>5$ & $19(16.4)$ & $3(13.6)$ & $16(17.0)$ & \\
\hline \multicolumn{5}{|l|}{ Anthropometry } \\
\hline WAZ, mean (SD) & $-2(2)$ & $-2(2)$ & $-2(2)$ & 0.432 \\
\hline WAZ categories, $n(\%)$ & & & & 0.513 \\
\hline Mild-normal & $55(47.4)$ & $8(36.4)$ & $47(50.0)$ & \\
\hline Moderate underweight & $31(26.7)$ & $7(31.8)$ & $24(25.5)$ & \\
\hline Severe underweight & $30(25.9)$ & $7(31.8)$ & $23(24.5)$ & \\
\hline HAZ, mean (SD) & $-2(2)$ & $-2(2)$ & $-2(1)$ & 0.364 \\
\hline HAZ categories, $n(\%)$ & & & & 0.587 \\
\hline Mild-normal & $65(60.7)$ & $11(55.0)$ & $54(62.1)$ & \\
\hline Moderate stunting & $23(21.5)$ & $6(30.0)$ & $17(19.5)$ & \\
\hline Severe stunting & $19(17.8)$ & $3(15.0)$ & $16(18.4)$ & \\
\hline WHZ, mean (SD) & $-1(2)$ & $-1(2)$ & $-1(2)$ & 0.926 \\
\hline WHZ categories, $n(\%)$ & & & & 0.428 \\
\hline Mild-normal & $75(70.8)$ & $12(60.0)$ & $63(73.3)$ & \\
\hline Moderate wasting & $17(16.0)$ & $5(25.0)$ & $12(14.0)$ & \\
\hline Severe wasting & $14(13.2)$ & $3(15.0)$ & $11(12.8)$ & \\
\hline \multicolumn{5}{|l|}{ Social/family, $n(\%)$} \\
\hline Mother alive & $109(94.0)$ & $22(100.0)$ & $87(92.6)$ & 0.187 \\
\hline Mother primary caregiver & $101(87.1)$ & $20(90.9)$ & $81(86.2)$ & 0.551 \\
\hline Mother on ART & 15/104 (14.4) & $3 / 21(14.3)$ & $12 / 83(14.5)$ & 0.984 \\
\hline Any maternal PMTCT prophylaxis & $41(38.7)$ & $7(31.8)$ & $34(40.5)$ & 0.458 \\
\hline Literate primary caregiver & $77 / 81(95.1)$ & 9/11 (81.8) & $68 / 70(97.1)$ & $0.087^{\S}$ \\
\hline Formal housing & $62(53.9)$ & $9(42.9)$ & $53(56.4)$ & 0.261 \\
\hline Household treatment support & $67 / 98(68.4)$ & $11 / 16(68.8)$ & $56 / 82(68.3)$ & 0.971 \\
\hline Accessing support grant & $61(55.5)$ & $16(80.0)$ & $45(50.0)$ & 0.015 \\
\hline
\end{tabular}

PHC clinics or hospitals. The median time between down-referral and presentation was 27 days (IQR 19 - 33). However, 11.4\% (12/105) presented $>8$ weeks after down-referral, with a median of 77 days (IQR 66 - 129) days between down-referral and presentation. At the point of down-referral, $19.8 \%$ of patients were either LTFU (11/116) or experienced possible treatment interruption (12/116).

Twelve months after down-referral, $81.0 \%$ (94/116) of the study cohort remained in care, $64.7 \%(75 / 116)$ at the designated PHC clinic and a further $16.4 \%(19 / 116)$ at other sites. One patient had died. The remaining $18.1 \%(21 / 116)$ were LTFU, $52.4 \%(11 / 21)$ at the point of down-referral, having not attended a single PHC clinic visit in the Western Cape. Of those LTFU, $42.9 \%(9 / 21)$ were $<12$ months of age at the time of ART initiation.

\section{Response to ART at designated down-referral clinics}

The 75 children who remained in care at the designated down-referral clinics showed sustained clinical and immunological response to ART over the 12 months of follow-up (Table 3).

The proportion of children with moderate to severe underweight for age declined steadily from $50.7 \%(38 / 75)$ at initiation to $8.0 \%$
$(6 / 75) 12$ months after down-referral $(p<0.001)$. The median CD4\% had increased from $17 \%$ (IQR $11-23$ ) at initiation to $31 \%$ (IQR 23 - 37) at down-referral $(p<0.001)$, and this was sustained over 12 months of follow-up in the PHC clinics. The percentage of children with severe immunodeficiency declined from $69.3 \%$ at initiation to $1.3 \% 12$ months after down-referral $(p<0.001)$. Similar rates of viral suppression were documented at downreferral $(50.7 \%, 38 / 75)$ and 12 months after down-referral $(54.7 \%$, $41 / 75$ ) ( $p=0.62$ ). However, if only those children with documented VLs are considered, rates of viral suppression were much higher both at down-referral $(86.4 \%, 38 / 44)$ and 12 months later $(75.9 \%$, 41/54).

Despite these markers of improvement, $14.7 \%$ (11/75) required at least one hospital admission during the first year of follow-up after down-referral.

\section{Predictors of retention in care 12 months after down-referral}

In univariate analysis, TB coinfection at the time of ART initiation was significantly less prevalent in children RIC at 12 months 
Table 2. Clinical, disease and management factors at ART initiation

\begin{tabular}{|c|c|c|c|c|}
\hline & Total $(N=116)$ & Not RIC $(n=22)$ & $\operatorname{RIC}(n=94)$ & $p$-value ${ }^{\star}$ \\
\hline \multicolumn{5}{|l|}{ Disease factors } \\
\hline WHO clinical stage, $n(\%)$ & & & & 0.977 \\
\hline 1 & $4(3.6)$ & $1(5.0)$ & $3(3.3)$ & \\
\hline 2 & $7(6.3)$ & $1(5.0)$ & $6(6.5)$ & \\
\hline 3 & $50(44.6)$ & $9(45.0)$ & $41(44.6)$ & \\
\hline 4 & $51(45.5)$ & $9(45.0)$ & $42(45.7)$ & \\
\hline CD4+ absolute count (cells/ $\mu \mathrm{L})$, median (IQR) & $585(249-1067)$ & $665(276-1149)$ & $561(249-1056)$ & 0.584 \\
\hline CD4\%, median (IQR) & $18(11-23)$ & $19(16-24)$ & $17(11-23)$ & 0.251 \\
\hline $\log _{10}$ VL (copies/mL), median (IQR) & $6(5-6)$ & $5(5-6)$ & $6(5-6)$ & 0.196 \\
\hline Severe immunodeficiency ${ }^{\dagger}, n(\%)$ & $79(68.7)$ & $13(59.1)$ & $66(71.0)$ & 0.280 \\
\hline \multicolumn{5}{|l|}{ Clinical factors, $n(\%)$} \\
\hline Up-to-date immunisation status & $86 / 100(86.0)$ & $14 / 18(77.8)$ & $72 / 82(87.8)$ & 0.267 \\
\hline TB treatment at initiation & $21(18.1)$ & $7(31.8)$ & $14(14.9)$ & 0.048 \\
\hline \multicolumn{5}{|l|}{ Clinical management factors } \\
\hline Highest level of care at RCWMCH, $n(\%)$ & & & & 0.726 \\
\hline Ward & $80(70.2)$ & $16(76.2)$ & $64(68.8)$ & \\
\hline ICU & $18(15.8)$ & $3(14.3)$ & $15(16.1)$ & \\
\hline $\mathrm{OHC}$ & $16(14.0)$ & $2(9.5)$ & $14(15.1)$ & \\
\hline Place of ART initiation, $n(\%)$ & & & & 0.970 \\
\hline Ward & $60(52.2)$ & $12(54.6)$ & $48(51.6)$ & \\
\hline ICU & $11(9.6)$ & $2(9.1)$ & $9(9.7)$ & \\
\hline $\mathrm{OHC}$ & $44(38.3)$ & $8(36.4)$ & $36(38.7)$ & \\
\hline Time from HIV diagnosis to ART initiation, median (IQR) & $42(14-184)$ & $28(13-154)$ & $42(14-199)$ & 0.805 \\
\hline Follow-up at RCWMCH $>6$ months, $n(\%)$ & $74(63.8)$ & $11(50.0)$ & $63(67.0)$ & 0.135 \\
\hline Suppressed VL prior to down-referral ${ }^{\ddagger}, n(\%)$ & $55(47.4)$ & $7(31.8)$ & $48(51.1)$ & 0.104 \\
\hline
\end{tabular}

Table 3. Longitudinal changes of the 75 children who remained in care at the designated down-referral clinics 12 months after down-referral

\begin{tabular}{|c|c|c|c|c|}
\hline & Initiation & At down-referral & $\begin{array}{l}6 \text { months after down- } \\
\text { referral }\end{array}$ & $\begin{array}{l}12 \text { months after down- } \\
\text { referral }\end{array}$ \\
\hline WAZ, mean (SD) & $-2(2)$ & $-1(1)$ & $0(1)$ & $0(1)$ \\
\hline \multicolumn{5}{|l|}{ WAZ categories, $n(\%)$} \\
\hline Mild-normal & $37(49.3)$ & $54(72.0)$ & $66(88.0)$ & $65(86.7)$ \\
\hline Moderate & $19(25.3)$ & $5(6.7)$ & $4(5.3)$ & $5(6.7)$ \\
\hline Severe & $19(25.3)$ & $3(4.0)$ & $1(1.3)$ & $1(1.3)$ \\
\hline Unknown & $0(0.0)$ & $13(17.3)$ & $4(5.3)$ & $4(5.3)$ \\
\hline \multicolumn{5}{|l|}{$\mathrm{CD} 4 \%$} \\
\hline Children, $n$ & 75 & 45 & 54 & 45 \\
\hline Median (IQR) & $17(11-23)$ & $31(23-37)$ & $33(26-39)$ & $32(27-35)$ \\
\hline \multicolumn{5}{|c|}{ Severe immunodeficiency ${ }^{*}, n(\%)$} \\
\hline Yes & $52(69.3)$ & $4(5.3)$ & $6(8.0)$ & $1(1.3)$ \\
\hline No & $23(30.7)$ & $41(54.7)$ & $48(64.0)$ & $49(65.3)$ \\
\hline Unknown & $0(0.0)$ & $30(40.0)$ & $21(28.0)$ & $25(33.3)$ \\
\hline \multicolumn{5}{|l|}{ Suppressed VL, $n(\%)$} \\
\hline Yes & $0(0.0)$ & $38(50.7)$ & $47(62.7)$ & $41(54.7)$ \\
\hline No & $67(89.3)$ & $6(8.0)$ & $13(17.3)$ & $13(17.3)$ \\
\hline Unknown/not done & $8(10.7)$ & $31(41.3)$ & $15(20.0)$ & $21(28.0)$ \\
\hline
\end{tabular}

after down-referral than those not RIC $(13.8 \%$ v. $33.3 \%$, RR 0.4 , 95\% CI 0.2 - 0.9), and the proportion of caregivers in receipt of a social grant at the time of ART initiation was significantly lower for children RIC at 12 months of ART than for those not RIC $(51.1 \% \mathrm{v}$. 78.9\%, RR 0.7, 95\% CI 0.5 - 0.9). However, in multivariable logistic regression modelling, neither factor retained significance. 


\section{Discussion}

The present study showed that for children who remained in care at the designated PHC clinics, clinical and immunological gains achieved on ART were sustained for at least 12 months after downreferral. The results were consistent with those from larger studies in similar cohorts. ${ }^{[13,14]}$ It has also shown important negative outcomes associated with the down-referral process, with $18.9 \%$ of the study cohort LTFU, $50.0 \%$ of whom never presented to PHC clinics, and a further $11.4 \%$ having $>8$ weeks between down-referral and presentation to PHC clinics.

Similar patterns of LTFU and treatment interruptions were reported in a recent study from Dora Nginza Hospital in Port Elizabeth, Eastern Cape Province. In that study, of 644 children receiving ART who were transferred to primary and community health clinics for continuation of care, $33 \%$ never arrived at the designated clinic after down-referral. ${ }^{[15]}$ Furthermore, among the 1582 children initiated on ART during the study period, $53 \%$ of all loss to follow-up occurred through unsuccessful down-referral. Finally, by searching the National Health Laboratory Service database, results for 62\% (131/211) of those lost to follow-up on down-referral were obtained, and only $5 \%(10 / 211)$ of them were within 18 months of down-referral. This suggests that while many children did re-engage in care, significant treatment interruptions were common.

Both these SA studies are in stark contrast to a study conducted in Thailand, in which of 168 children down-referred from a tertiary hospital to several community hospitals, none were LTFU during a median follow-up time of 18 months. ${ }^{[16]}$ This perfect retention in care can be attributed to the intensive support and adherence counselling provided after down-referral, with ongoing involvement of the referral team, who provided active mentorship and clinical support to the treating teams in the community hospitals as well as 6-monthly clinical reviews of the down-referred children at the tertiary referral hospital. ${ }^{[16]}$ In contrast, the support available in our study was much less intensive, consisting of telephonic support for clinicians caring for HIV-infected children at the PHC clinics, and monthly clinical mentoring visits by an infectious diseases subspecialist from RCWMCH.

Another difference between our study and the Thai study is the age of the children. In the Thai study, the median age at initiation was 8.6 years, compared with 11 months for our cohort. In a study of $>5000$ infants ( $<12$ months of age) initiated on ART in southern Africa, the 3-year mortality and LTFU estimates were $16 \%$ and $29 \%$, respectively. ${ }^{[13]}$ These results suggest that HIV-infected infants are extremely vulnerable during the initial period on ART. More than $50 \%$ of the down-referred children in our study were infants at ART initiation, and this may partly explain the high prevalence of LTFU. In the study from Dora Nginza Hospital, however, the median age at ART initiation was 4 years and the median age at transfer 8 years, suggesting that age alone is an insufficient explanation for the high prevalence of LTFU.

Death may have accounted for some of the children designated as LTFU in our study. In a study of the outcome of $>3000$ HIVinfected children treated with ART in four SA provinces, linkage to the national death registry confirmed mortality of $34 \%$ of 68 children with valid identity numbers. ${ }^{[17]}$

Another factor that may have contributed to LTFU in our study is the movement of patients between treating clinics and other parts of SA without the knowledge of the attending clinicians. While those who continued care in the Western Cape should have been identified through the search of the provincial database, the lack of a national unique patient identifier prohibits the tracing of patients who continue care in other provinces. In our study, within 12 months of down-referral, $1.7 \%$ of children had a documented transfer to another province. In the Dora Nginza hospital study, 18\% of those who successfully transferred had a subsequent transfer to another facility during the study period. These figures show that many children receiving ART may experience multiple transfers between treatment facilities, with or without the knowledge of treating physicians.

The results of our study and the Dora Nginza Hospital study suggest that the process of transitioning care from one site to another is inherently vulnerable. However, evidence to guide down-referral criteria or to assist in identifying those children at high risk of LTFU is severely limited. Although clinical stability is reported as a down-referral criterion, those down-referred in this study included children recently initiated on ART, children with unsuppressed VLs, and children who had defaulted from ART while in care at the OHC. Documented viral suppression was not associated with increased retention in care. In the Dora Nginza study, $16 \%$ of transferred children had VLs $>1000$ copies $/ \mathrm{mL}$. Importantly, in the study by Morsheimer et al. ${ }^{[10]} 77 \%$ of patients down-referred with unsuppressed VLs achieved virological suppression within 6 months of treatment at the PHC clinic, suggesting improved adherence possibly resulting from reduced barriers to accessing care. This raises further doubt about the validity of 'clinical stability' as a downreferral criterion.

Unfortunately, our study did not identify any specific factors associated with successful down-referral. Although the primary caregiver accessing a support grant and coexistent TB disease at the time of ART initiation were both associated with non-retention in care, neither was significant in multivariable models and it is difficult to understand the relevance of this finding. The Dora Nginza study also failed to identify risk factors for unsuccessful downreferral, but in the study of adolescent transfer in the Western Cape, virological suppression was associated with successful transfer. ${ }^{[15]}$ While documented VL suppression was not associated with retention in care in our study, it would be important to assess this in larger cohorts of similarly aged young children.

Until further evidence allows us to stratify risk of LTFU at down-referral, clinicians should regard this transition of care as a vulnerable point in the management of children living with HIV. Greater attention should be given to monitoring down-referred children and strengthening the support provided to them and their caregivers. While the level of support given to down-referred children in the Thai study ${ }^{16]}$ is perhaps unrealistic in the SA context, more formalised communications with referral sites and telephonic contact tracing of patients after down-referral is feasible. Furthermore, community adherence support has been shown to improve retention in care of children on ART in the SA public sector and should be considered for caregivers of children down-referred to community clinics on ART. ${ }^{[18]}$

\section{Study strengths and limitations}

This is one of the first SA studies that has followed a cohort of children receiving ART at a tertiary referral hospital through the process of down-referral to PHC clinics and evaluated their clinical responses during the first year after down-referral. It provides a unique perspective on the impact of the down-referral process on paediatric ART provision and care under routine operational conditions.

There are a number of limitations to this study. The main limitation is the lack of a comparator group who were not down-referred, preventing comparison of outcomes between these two groups. The small cohort size may also have limited the ability to detect significant factors affecting retention in care. Furthermore, the study 
only looked at two of the PHC clinics that fall into the RCWMCH drainage area, so the results are not necessarily generalisable. Finally, the study design did not allow for collection of data at PHC clinics other than the two designated referral clinics. Response to ART after down-referral therefore could not be assessed for those who continued care at other sites.

\section{Conclusions}

This study has shown that the greatest risk of LTFU and treatment interruption associated with down-referral in provision of paediatric ART occurs at the point of down-referral. Children who successfully navigate this transition in care show sustained clinical and virological improvements.

Acknowledgements. We are grateful to Dr Tony Hawkridge, Western Cape Department of Health, for permission to search the provincial electronic databases for ART data on a subset of patients and to Dr Nicki Tiffin and Prof. Andrew Boulle of the Western Cape Provincial Health Data Centre for assistance with the database searches.

Author contributions. JC drafted the study protocol, collected and analysed the data, and drafted the manuscript. PA assisted with extraction of data from the RCWMCH database and patient folders. M-AD contributed to the data extraction, data analysis and drafting of the manuscript. BE conceived the study and participated in the data analysis and drafting of the manuscript. All authors read and approved the final manuscript.

Funding. $\mathrm{M}-\mathrm{AD}$ and $\mathrm{BE}$ receive funding from the National Institutes of Health for the International Epidemiologic Databases to Evaluate AIDS Southern Africa research collaboration (grant no. U01AI069924).

Conflicts of interest. None. 1. Newell ML, Coovadia H, Cortina-Borja M, Rollins N, Gaillard P, Dabis F. Mortality of infected
and uninfected infants born to HIV-infected mothers in Africa: A pooled analysis. Lancet 2004;364(9441):1236-1243, https://doi.org/10.1016/S0140-6736(04)17140-7
2. Innes S, Lazarus E, Otwombe K, et al. Early severe HIV disease precedes early antiretroviral therapy
in infants: Are we too late? J Int AIDS Soc 2014:17(1):18914, https://doi.org/10.7448/IAS.17.1.18914 UNAIDS. 2015 Progress report on the global plan tow among children and keeping their mothers alive. 26 November 2015. http.//www infections among children and keeping their mothers alive. 26 November 2015. http://www.unaids.org/en/

4. National Department of Health. The South African Antiretroviral Treatment Guidelines 2013, http. National Department of Health. The South African Antiretroviral Treatment Guidelines 2013. http://
www.sahivsoc.org/Files/2013\%20ART\%20Treatment\%20Guidelines\%20Final\%2025\%20March\%20 www.sahivsoc.org/Files/2013\%20ART\%20Treatmen

5. Zachariah R, Ford N, Philips M, et al. Task shifting in HIV/AIDS: Opportunities, challenges and proposed actions for sub-Saharan Africa. Trans R Soc Trop Med Hyg 2009;103(6):549-558. https://doi. proposed actions for sub-Saharan $A$.
org/10.1016/j.trstmh.2008.09.019

6. Kredo T, Ford N, Adeniyi FB, Garner P. Decentralising HIV treatment in lower- and middle-income countries (Review). Cochrane Database Syst Rev 2013, Issue 6. Art. No.: CD009987. https://doi. org/10.1002/14651858.CD009987.pub2

7. Eley B, Nuttall J. Antiretroviral therapy for children: Challenges and opportunities. Ann Trop Paediatr Int Child Health 2007;27(1):1-10. https://doi.org/10.1179/146532807X170448

8. Johnson L. Children count - children starting ART. http://www.childrencount.ci.org.za/indicator. php?id=5\&indicator $=20$ (accessed 11 July 2012).

9. Davies MA, Tsondai P, Tiffen N, et al. Where do HIV-infected adolescents go after transfer? Tracking transition/transfer of HIV-infected adolescents using linkage of cohort data to the health information system platform. J Int AIDS Soc 2017;20(Suppl 3):16-24. https://doi.org/10.7448/IAS.20.4.21668

10. Morsheimer MM, Dramowski A, Rabie H, Cotton MF. Paediatric ART outcomes in a decentralised model of care in Cape Town, South Africa. South Afr J HIV Med 201415(4):148-153. https://doi. org/10.4102/sajhivmed.v15i4.332

1. Kuczmarski RJ, Ogden CL, Grummer-Strawn LM, et al. CDC growth charts: United States. Adv Data 2000;314(314):1-27.

12. World Health Organization. WHO Case Definitions of HIV for Surveillance and Revised Clinical Staging and Immunological Classification of HIV-Related Disease in Adults and Children. Geneva: WHO, 2007. http://www.who.int/hiv/pub/guidelines/hivstaging/en/ (accessed 22 February 2016).

13. Technau K-G, Schomaker M, Kuhn L, et al. Virologic response in children treated with abacavircompared with stavudine-based antiretroviral treatment: A South African multi-cohort analysis. Pediatr Infect Dis J 2014;33(6):617-622. https://doi.org/10.1097/INF.0000000000000222

14. Porter M, Davies M, Mupani MK, et al. Outcomes of infants starting antiretroviral therapy in southern Africa, 2004-2012. J Acquir Immune Defic Syndr 2015;69(5):593-601 https://doi.org/10.1097/ qai. 0000000000000683

15. Teasdale CA, Sogaula N, Yuengling KA, et al. High risk of loss to follow-up among South African children on ART during transfer, a retrospective cohort analysis with community tracing. J Int AIDS Soc 2017;20(1):21748. https://doi.org/10.7448/IAS.20.1.21748

16. Hansudewechakul R, Naiwatanakul T, Katana A, et al. Successful clinical outcomes following decentralization of tertiary paediatric HIV care to a community-based paediatric antiretroviral decentralization of tertiary paediatric HIV care to a community-based paediatric antiretroviral
treatment network, Chiangrai, Thailand, 2002 to 2008. J Int AIDS Soc 2012;15(2):17358. https://doi. treatment network, Chiangr
org/10.7448/IAS.15.2.17358

17. Fatti G, Bock P, Eley B, Mothibi E, Grimwood A. Temporal trends in baseline characteristics and Fatti G, Bock P, Eley B, Mothibi E, Grimwood A. Temporal trends in baseline characteristics and
treatment outcomes of children starting antiretroviral treatment: An analysis in four provinces in treatment outcomes of children starting antiretroviral treatment: An analysis in four provinces in
South Africa, 2004 - 2009. J Acquir Immune Defic Syndr 2011;58(3):e60-e67. https://doi.org/10.1097/ South Africa, 2004 - 2009 ,

18. Grimwood A, Fatti G, Mothibi E, Malahlela M, Shea J, Eley B. Community adherence support improves programme retention in children on antiretroviral treatment: A multicentre cohort study in South Africa. J Int AIDS Soc 2012;15(2):17381. https://doi.org/10.7448/IAS.15.2.1738 